\title{
FATAL CASES OF DISSEMINATED NOCARDIOSIS: CHALLENGES TO PHYSICIANS AND CLINICAL MICROBIOLOGISTS - CASE REPORT
}

\author{
KlÁra PiUKOVICS ${ }^{1}$, ViKTÓRIA BERTALAN ${ }^{2}$, GABriella TERHES ${ }^{3}$, \\ ÁGNES BÁTHORI $^{4}$, EdIT HAJdU' ${ }^{5}$, GYUlA POKORNY ${ }^{6}$, LÁSZló KOVÁCS ${ }^{6}$ \\ and EDIT URBÁN ${ }^{3 *}$ \\ ${ }^{1}$ Department of Haematology, Second Department of Medicine and Cardiology Center, \\ University of Szeged, Szeged, Hungary \\ ${ }^{2}$ Intensive Care Unit, Department of Anaesthesiology and Intensive Therapy, University \\ of Szeged, Szeged, Hungary \\ ${ }^{3}$ Institute of Clinical Microbiology, University of Szeged, Szeged, Hungary \\ ${ }^{4}$ Department of Pathology, University of Szeged, Szeged, Hungary \\ ${ }^{5}$ Department of Infectious Diseases, First Department of Medicine, University of Szeged, \\ Szeged, Hungary \\ ${ }^{6}$ Department of Rheumatology, University of Szeged, Szeged, Hungary
}

(Received: 2 February 2016; accepted: 11 July 2016)

Despite the development in the identification of Nocardia spp., common challenges exist in the laboratory diagnosis and management of nocardiosis. We report two cases of disseminated nocardiosis in a patient with hematologic disorder and in a patient with systemic lupus erythematosus, where the cooperation between various specialists was essential to set up the adequate diagnosis of disseminated nocardiosis.

Keywords: Nocardia spp., immunocompromised, fatal, culture, infection

\section{Introduction}

Nocardia species are ubiquitous environmental filamentous bacteria that may cause various infections in humans [1]. The incidence of these infections has increased among immunocompromised patients in the last three decades, because of widespread use of immunosuppressive therapy and modern microbiological diagnostic procedures. In spite of the increasing number of these infections and the development in identification of Nocardia spp., the laboratory diagnosis and

\footnotetext{
*Corresponding author; E-mail: urban.edit@med.u-szeged.hu
} 
management of nocardiosis are quite difficult, and probably nocardiosis is underdiagnosed [2]. Development of infection is mainly due to inhalation or cutaneous inoculation of this pathogen. Nosocomial infection is rarely detected. Several conditions predispose to nocardiosis, such as corticosteroid therapy, malignant hematological diseases, chronic pulmonary disease, and bone marrow and solid organ transplantations. In immunocompromised host, hematogenous dissemination, involving the central nervous system (CNS) and skin, is characteristic. In this patient group, morbidity and mortality are high because of the patient's impaired cell-mediated immunity and frequent antibiotic resistance of the isolated strain [3].

\section{Presentation of Cases}

\section{Case 1}

A 37-year-old man was diagnosed with large granular lymphocytic leukemia in 1998. He underwent six cycles of combined chemotherapeutic treatment (cytoxan, vincristine, and prednisolone), and later had long-term steroid therapy due to Coombs positive hemolytic anemia and active hemolytic events. He was hospitalized due to several febrile episodes 6 months before the present admission, but infectious agents could not be detected. Upon admission, the patient complained of generalized fatigue, fever, and vomiting. On physical examination, the patient's blood pressure was 130/90 $\mathrm{mmHg}$ and his heart rate was 104 beats/min. After hospital admission, headache and dizziness started. Neurological examination did not reveal any signs of meningitis. Serum laboratory results showed normal level of procalcitonine, liver and kidney function, elevated leukocyte count $(34.2 \mathrm{~g} / \mathrm{L})$ with $80 \%$ neutrophil cells, mild anemia (hemoglobin level $11.3 \mathrm{~g} / \mathrm{L}$ ), and normal platelet count $(339 \mathrm{~g} / \mathrm{L})$. Three days later, computed tomography (CT) was done because of central type paresis of facial and hypoglossal nerves. The CT scan showed multiple lesions $(6-13 \mathrm{~mm}$ in diameter) with perifocal edema. Stereotactic core biopsy from lesions could not be performed because of deterioration in the patient's clinical status. Blood and urine samples for bacteriological culture were collected, and empiric antibiotic therapy (imipenem-cilastatin $500 \mathrm{mg}$ every $6 \mathrm{~h}$ ) was started. Two blood culture bottles gave positive signal after 52 and $65 \mathrm{~h}$ of incubations, and in the Gram-stained preparations, Gram-positive branching filaments could be observed (Figure 1A). After $24 \mathrm{~h}$ of incubation in $5 \% \mathrm{CO}_{2}$ atmosphere, small colonies were grown. Because of unsuccessful identification of the isolated strains using commercial kit (VITEK 2 GP ID card, bioMérieux, Marcy-l'Étoile, France), 16S rDNA sequencing was performed. Nucleic acid from 


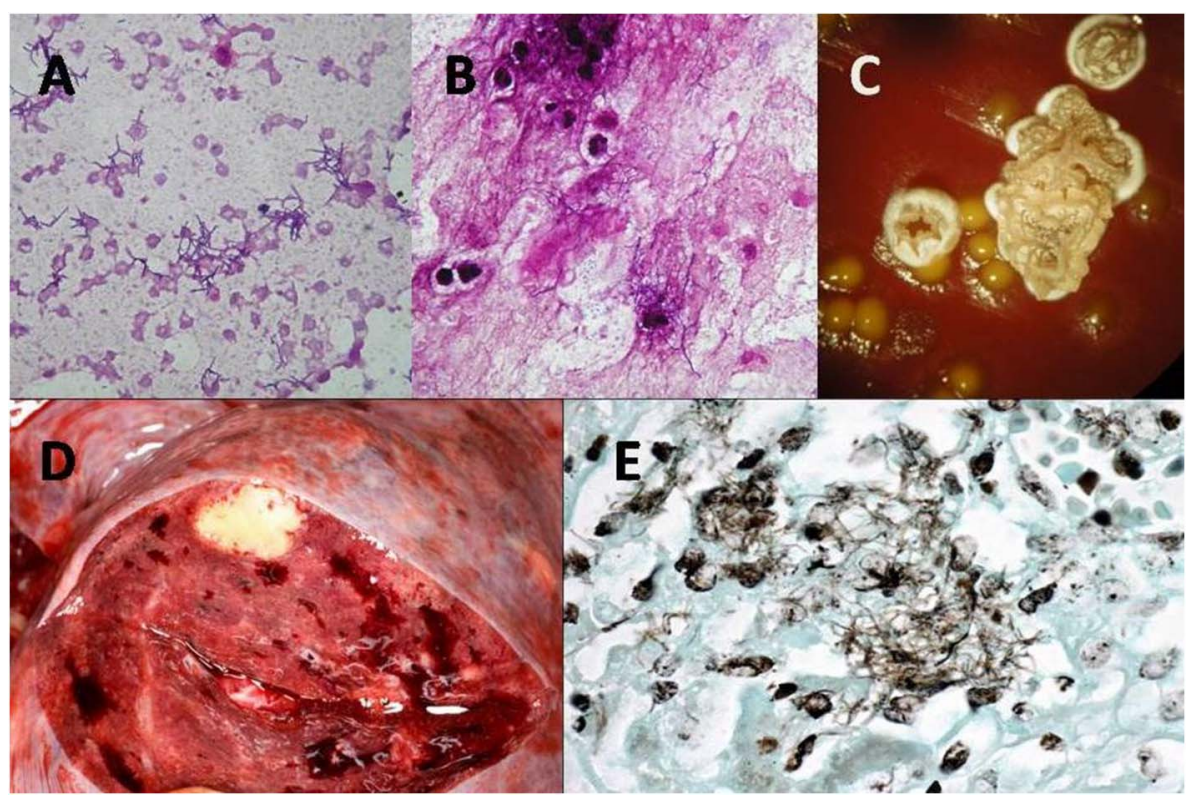

Figure 1. (A) Gram-positive branching filaments from blood culture (1,000× magnification); (B) Gram-positive branching filaments from endotracheal aspirate (1,000× magnification); (C) $N$. farcinica (small colonies) and $N$. cyriacigeorgica (white colonies) could be cultured from endotracheal aspirate; (D) subpleural abscess (diameter $2 \mathrm{~cm}$ ) in the lung; (E) Grocott staining of the pulmonary abscess demonstrates branching bacterial filaments, corresponding to Nocardia infection (original magnification, 40x)

the colonies was purified using QIAamp DNA mini kit (Qiagen) and the 16S rDNA was amplified using previously published primers (E8F and E533R) [4]. On the basis of sequence analysis of the amplified polymerase chain reaction (PCR) product, Nocardia farcinica was identified. The patient died due to septic episode 2 days later and autopsy was not performed.

\section{Case 2}

A 21-year-old man with systemic lupus erythematosus (SLE) was hospitalized in the Clinics of Rheumatology because of fever, cough, and dyspnea. The disease had been diagnosed on the basis of arthralgias, anemia, leucopenia, proteinuria, low $\mathrm{C} 3$ complement level, anti-dsDNA antibody positivity, and biopsy proven diffuse-global active lupus glomerulonephritis 3 months before the present admission. Methylprednisolone was given ( $250 \mathrm{mg} /$ day intravenously), and dosage was gradually reduced to $16 \mathrm{mg} /$ day after achieving remission. Shortly 
after the present admission, the patient was transferred to the ICU because of multi-organ failure, bilateral pneumonia, and suspicion of lung abscess. Empiric intravenous antibiotic (4.5 g of piperacillin/tazobactam 3 times/day, $1 \mathrm{~g}$ of amikacin daily, and $1 \mathrm{~g}$ of vancomycin twice/day), and antifungal (amphotericin B $50 \mathrm{mg}$ /day) therapies were started after sample collection for microbiological culture (blood culture and endotracheal aspirate). Laboratory results showed elevated white blood cell $(14.09 \mathrm{~g} / \mathrm{L}$ with $90 \%$ neutrophil granulocytes) and C-reactive protein $(327 \mathrm{mg} / \mathrm{L})$. In the Gram-stained preparation of the endotracheal specimens, Gram-positive branching filaments with the presence of granulocytes were observed (Figure 1B). On the basis of this finding, antibiotic therapy was completed with trimethoprim/sulfhamethoxazole. After $72 \mathrm{~h}$ of incubation, small and white colonies were cultured (Figure 1C). Because of unsuccessful use of commercial identification system (VITEK 2 GP ID card, bioMérieux), DNA was isolated from two types of colonies, and 16S rDNA sequencing was performed using the above-mentioned primers. On the basis of the sequence of the amplified PCR products, in case of small colonies, N. farcinica, whereas in case of white colonies, Nocardia cyriacigeorgica were identified. Vasopressor therapy and mechanical ventilation were introduced because of hypotension and respiratory failure. His renal function was worsened, thus renal dialysis was started. On the basis of antimicrobial susceptibility testing (E-test, AB Biodisk, Solna, Sweden), N. farcinica and N. cyriacigeorgica were susceptible to imipenem and amikacin; thus, antibiotic therapy was changed to imipenem-amikacin combination. Blood culture bottles also gave positive signal after $52 \mathrm{~h}$ of incubation, and $N$. farcinica with the same susceptibility pattern was grown from the blood. During the antibiotic therapy, the number of bacteria in the endotracheal aspirates was reduced from $>10^{5} \mathrm{CFU} / \mathrm{mL}$ to $10^{2} \mathrm{CFU} / \mathrm{mL}$ in the case of $N$. farcinica, whereas $N$. cyriacigeorgica was not detected from the subsequent respiratory specimens. After temporary improvement in the patient's condition, deteriorations in SLE and adult respiratory distress syndrome (ARDS) have been observed again. Following a 19day stay in ICU, the patient died due to multi-organ failure.

The autopsy demonstrated multiple, $0.5-1.5 \mathrm{~cm}$ sized abscesses in the lungs (Figure 1D), mainly at the periphery, and an abscess of $4 \mathrm{~cm}$ in diameter in the right upper lobe. The kidneys were abnormally swollen and 4-6 $(0.5-0.8 \mathrm{~mm}$ sized) abscesses were encountered in the cortex. Histologically, the abscesses were characterized by widespread suppuration and necrosis, a few epithelioid cells at the margins and the absence of a pyogenic membrane. The Grocott staining revealed long, thin, and beaded bacterial filaments, sometimes branching in right angles (Figure 1E). In the areas neighboring pulmonary abscesses, diffuse alveolar damage with hyaline membranes was noted. The findings corresponded to a Nocardia infection of the lungs and kidneys, and ARDS. Since the activity lesions 
of lupus glomerulonephritis had almost disappeared, the therapy of lupus nephritis proved to be effective. The cause of deterioration of the patient's status was the Nocardia infection, not controlled efficiently by the antibiotic treatment.

\section{Conclusions}

Nocardiosis is most frequently described as opportunistic infection in immunocompromised patients, especially, in patients with depressed cell-mediated immunity. Pulmonary nocardiosis is the most common clinical presentation of this infection, while among extrapulmonary forms, CNS involvement is quite common $[1,5,6]$. Bacteremia caused by Nocardia spp. is rarely described clinical entity, and in the majority of patients, concurrent pulmonary (64\%), cutaneous (28\%), and CNS involvements (19\%) could be observed [6]. In the case of disseminated nocardiosis, two or more organs are infected, and in the majority of cases, due to airway transmission, the lung is also affected. In case 1, this could not be confirmed radiologically and the patient did not have any respiratory symptoms, whereas in case 2 , the patient had severe respiratory symptoms and later autopsy confirmed nocardial infection of lungs and kidneys. Several publications showed case reports about the presence of $N$. farcinica in various infections, but according to Christidou et al. [7] until 2004, only 11 English publications were found where $N$. farcinica bacteremia was described.

During the last 20 years, because of the development in microbiological methods, several changes could be observed in the taxonomy of genus Nocardia, thus we can get more adequate data about the prevalence and the role of certain species in various clinical pictures. On the basis of literature data, N. farcinica and $N$. cyriacigeorgica are increasingly reported in human infections [5]. These species are associated with pulmonary and extrapulmonary forms of nocardiosis; in addition to these, $N$. farcinica has emerged as a nosocomial pathogen, while the detection of $N$. farcinica from blood is rare [5, 8]. Christidou et al. [7] reported a case of fatal $N$. farcinica bacteremia in a patient with lung cancer, and they found only 11 previously reported cases, in English literature. The majority of patients were male and had one or more predisposing factors, and the lung was the most common primary site of infection. The mortality rate was $41.7 \%$; similarly, poor outcome associated with $N$. farcinica was previously reported by Torres et al. [9]. General treatment recommendations for nocardiosis are difficult to establish due to the lack of controlled trials and variable in vitro antibiotic susceptibility patterns [6]. In addition to these, it is difficult to perform antimicrobial susceptibility testing because of the slow growing nature of several Nocardia spp., inoculum consistency, and interpretation of cutoff [10]. The treatment of $N$. farcinica infections is 
also problematic, because the majority of clinical isolates are multi-resistant; thus, in our case, the isolated strain was only susceptible to imipenem and amikacin.

Here, we have described two cases of nocardiosis in immunocompromised hosts. In the first case, we supposed that multiple lesions caused by Nocardia spp. on the basis of CT scan, signs, and blood culture isolate. Peculiarity of the second case was that two Nocardia spp. could be isolated from the respiratory tract, and in spite of the antibiotic susceptibility results, combination therapy did not cure the infection. Owing to special culture condition of Nocardia spp., communication between specialists is essential to successful detection of these pathogens.

\section{Conflict of Interest}

The authors declare that they have no conflict of interest.

\section{References}

1. Brown-Elliott, B., Brown, J. M., Conville, P. S., Wallace, R. J., Jr.: Clinical and laboratory features of the Nocardia spp. based on current molecular taxonomy. Clin Microbiol Rev 19, 259-282 (2006).

2. Corti, M. E., Villafane-Fioti, M. F.: Nocardiosis: A review. Int J Infect Dis 7, 243-250 (2003).

3. Saubolle, M. A., Sussland, D.: Nocardiosis: Review of clinical and laboratory experience. J Clin Microbiol 41, 4497-4501 (2003).

4. Baker, G. C., Smith, J. J., Cowan, D. A.: Review and re-analysis of domain-specific $16 \mathrm{~S}$ primers. J Microbiol Methods 55, 541-555 (2003).

5. Valerio Minero, M., Marín, M., Cercando, E., Rabadán, M. P., Bouza, E., Munoz, P.: Nocardiosis at the turn of the century. Medicine 88, 250-261 (2009).

6. Wilson, J. W.: Nocardiosis: Updates and clinical overview. Mayo Clin Proc 87, 403-407 (2012).

7. Christidou, A., Maraki, S., Scoulica, E., Mantadakis, E., Agelaki, S., Samonis, G.: Fatal Nocardia farcinica bacteremia in a patient with lung cancer. Diagn Microbiol Infect Dis 50, 135-139 (2004).

8. Schaal, K. P., Lee, H. J.: Actinomycete infections in humans - A review. Gene 115, 201211 (1992).

9. Torres, O. H., Domingo, P., Pericas, R., Boiron, P., Montiel, J. A., Vazquez, G.: Infection caused by Nocardia farcinica: Case report and review. Eur J Clin Microbiol Infect Dis 19, 205-212 (2000).

10. Schlaberg, R., Fisher, M. A., Hanson, K. E.: Susceptibility profiles of Nocardia isolates based on current taxonomy. Antimicrob Agents Chemother 58, 795-800 (2014). 